\title{
Estimation of Minimal Clinically Important Difference in EQ-5D Visual Analog Scale Score After Pulmonary Rehabilitation in Subjects With COPD
}

\author{
Andrea Zanini MD, Marina Aiello MD, Daniela Adamo MD, Silvia Casale MD, \\ Francesca Cherubino PhD, Sabrina Della Patrona MD, Eleonora Raimondi MD, \\ Elisabetta Zampogna MSc, Alfredo Chetta MD, and Antonio Spanevello MD
}

\begin{abstract}
BACKGROUND: The effect of pulmonary rehabilitation (PR) on the EuroQol Group's 5-dimension questionnaire (EQ-5D) in COPD has been poorly investigated. In addition, conflicting results were reported about the visual analog scale component of EQ-5D (EQ-VAS). The purpose of this study was to evaluate the responsiveness of EQ-VAS to PR and its relationship with clinical and functional parameters in subjects with COPD, as well as to define the minimal clinically important difference (MCID) estimate for the EQ-VAS after PR. METHODS: A total of 468 in-patients with stable moderate-to-severe COPD, allocated to a 3-wk PR program, were retrospectively evaluated. EQ-VAS was assessed before and after PR, and its relationship with baseline pulmonary function, changes in 6-min walk test, and baseline, and transitional dyspnea index (BDI/TDI) after PR were evaluated. Using an anchor-based approach and receiver operating characteristic curves, the EQVAS change cutoff that identified subjects achieving the known MCID for TDI after PR was identified. RESULTS: Four hundred and thirty-nine subjects (94\%, mean FEV $55.3 \%$ predicted) completed pre- and post-PR EQ-VAS scores. After PR, EQ-VAS increased from $58 \pm 17$ to $72 \pm 15$ $(\triangle E Q-V A S 14 \pm 12, P<.001) . \triangle E Q-V A S$ was negatively related to baseline $\mathrm{FEV}_{1}(r=-0.32$, $P<.001)$ and positively to TDI $(\mathrm{r}=0.50, P<.001)$ and 6 -min walk distance $(\mathrm{r}=0.46, P<.001)$ changes. Receiver operating characteristic curves identified an EQ-VAS change cutoff of 8 as the best discriminating value to identify the MCID for TDI ( 0.78 sensitivity and 0.81 specificity; area under curve: $0.845, P<.001)$. CONCLUSIONS: Our study shows that, in in-patients with stable moderate-to-severe COPD, EQ-VAS is a valid and reliable tool to assess the responsiveness to PR, with an estimated MCID of 8 points. The EQ-VAS can be a practical alternative to more timeconsuming measures of health-related quality of life. Key words: EuroQol; VAS; pulmonary rehabilitation; COPD. [Respir Care 2015;60(1):88-95. ( 2015 Daedalus Enterprises]
\end{abstract}

\section{Introduction}

Pulmonary rehabilitation (PR) is a widely used and comprehensive intervention, which promotes improvements in

Drs Zanini, Adamo, Casale, Cherubino, Dell Patrona, Raimondi, Zampogna, and Spanevello are affiliated with the Division of Pneumology, Istituto di Ricovero e Cura a Carattere Scientifico Rehabilitation Institute of Tradate, Salvatore Maugeri Foundation, Tradate; Drs Zanini and Spanevallo are affiliated with the Department of Clinical and Experimental Medicine, University of Insubria, Varese; Drs Aiello and Chetta are affiliated with the Respiratory Disease and Lung Function Unit, Department of Clinical and Experimental Medicine, University of Parma, Parma, Italy. exercise capacity, symptoms, and quality of life in patients with COPD who have clinical and functional impairment. ${ }^{1}$ The EuroQol Group's 5-dimension questionnaire (EQ-5D), a generic preference-based instrument, is increasingly used

\footnotetext{
The authors have disclosed no conflicts of interest.

Correspondence: Andrea Zanini MD, Divisione di Pneumologia, Fondazione Salvatore Maugeri, Istituto di Ricovero e Cura a Carattere Scientifico, Via Roncaccio, 16-21049 Tradate, Italy. E-mail: andrea.zanini@ fsm.it.
}

DOI: $10.4187 /$ respcare.03272 
as a global measure of health-related quality of life (HRQOL). ${ }^{2}$ EQ-5D comprises a 5-dimension (mobility, self-care, usual activities, pain/discomfort, and anxiety/ depression) questionnaire and a $20-\mathrm{cm}$ visual analog scale (EQ-VAS) ranging from 0 (worst imaginable health) to 100 (best imaginable health), along which the respondents rate their health status. Several studies measured HRQOL, by means of EQ-5D, in patients with COPD during stable phases of the disease ${ }^{3-6}$ and during exacerbations. ${ }^{7-9}$ In stable disease, the EQ-5D's ability to discriminate between GOLD stages has been shown for total score ${ }^{3,4}$ and EQVAS, ${ }^{5}$ but ceiling effects have also been reported for EQVAS. ${ }^{4,10} \mathrm{Up}$ to now, only one study has examined the responsiveness of EQ-5D after PR in patients with COPD, ${ }^{10}$ showing a significant improvement in EQ-5D utility score and a trend toward significant increase in EQ-VAS.

HRQOL questionnaires, even if self-administered, often require operator supervision, with unavoidable expenditure in terms of time. It is necessary to identify simple and quick instruments that give reliable health and quality of life outcomes and that can be performed in most clinical settings, including rehabilitation centers, and are suitable for the largest number of subjects. The EQ-VAS is a practical and quick standardized instrument, able to provide an estimate of HRQOL, sufficiently precise and very sensitive to changes. ${ }^{11}$ Our group has recently reported a significant increase in EQ-VAS after a PR program in a large cohort of subjects with COPD with co-morbidities. ${ }^{12}$ However, no study has been specifically conducted to point out the value of VAS scores in subjects with COPD.

The first aim of this study was to examine the responsiveness of EQ-VAS to PR in subjects with clinically stable COPD, as well as its relationship with functional baseline parameters and PR outcomes. The second aim was to establish the minimal clinically important difference (MCID) for EQ-VAS after PR in subjects with COPD. Finally, we intended to ascertain whether baseline lung function and PR outcomes could predict a benefit in terms of a significant change in EQ-VAS after PR. Therefore, we performed a retrospective analysis from a database of in-patients with COPD who had undergone PR in a tertiary healthcare center.

\section{Methods}

\section{Design of the Study}

A retrospective analysis was performed on data collected from subjects with COPD admitted to our rehabilitation center from January 1, 2009 to December 31, 2012. Spirometry and blood gas analysis together with walking capacity, dyspnea, and HRQOL were obtained in all subjects at admission and at completion of the PR program. On day 1, subjects received a medical evaluation (medical

\section{QUICK LOOK}

\section{Current knowledge}

Pulmonary rehabilitation (PR) is a comprehensive intervention, which promotes improvements in exercise capacity, symptoms, and quality of life in patients with COPD with clinical and functional impairment. The EuroQoL Group's 5-dimension questionnaire (EQ-5D) is a generic preference-based instrument, frequently used as a global measure of health-related quality of life (HRQOL).

\section{What this paper contributes to our knowledge}

In in-patients with stable moderate-to-severe COPD, the EQ-5D visual analog scale (EQ-VAS) was a valid and reliable tool to assess response to PR. The EQ-VAS can be a practical alternative to more time-consuming measures of HRQOL.

history, physical examination, and vital signs), pulmonary function tests, and blood gas analysis. On the subsequent day, subjects completed BDI and EQ-VAS measurements and performed a 6-min walk test (6MWT). On day 3, subjects started the PR program. The last day before discharge, subjects performed final assessments. Correlation between baseline variables and improvement in HRQOL were also analyzed. Finally, the MCID for EQ-VAS after PR was estimated.

\section{Subjects}

We examined 497 subjects with COPD who attended an in-patient PR program. All subjects had a diagnosis for COPD according to the GOLD criteria. ${ }^{13}$ Subjects who had an exacerbation over the previous 4 wk were excluded, as well as subjects who were not able to perform the 6MWT. Subjects who did not complete the PR program, for intercurrent COPD exacerbation, or any unstable medical condition, were also excluded. Contraindications for participation in the PR program included musculoskeletal disorders, malignant diseases, unstable cardiac condition, and lack of adherence to the program. A total of 468 subjects were considered eligible for the study.

The Charlson index, a method for classifying comorbid conditions that might alter the risk of mortality, assigns a score to each disease that is proportional to the diseaserelated risk of death from the individuals' self-reported comorbidities. ${ }^{14}$ For each admitted subject, the Charlson index was computed by the physician, and the obtained value was not adjusted for age and did not include COPD in the individual's score. Diagnostic confirmation was in- 
directly assessed by means of chart review, biochemical data, and specific procedures that were performed during the hospitalization.

All subjects were receiving regular pharmacologic treatment (tiotropium in 431 subjects, inhaled long-acting $\beta_{2^{-}}$ agonists in 391 subjects, and inhaled corticosteroids in 329 subjects). One hundred and twenty subjects were under long-term oxygen therapy. One hundred and sixteen subjects out of 468 had 2 or more exacerbations in the previous year and were classified as frequent exacerbators, to ascertain whether they differed in PR outcomes as compared with the remaining subjects.

In all subjects, the clinical and functional assessment had been undertaken for clinical reasons at the request of the subject's clinician. The data used in the study are related to the subjects who gave their consent to the use of data for research purposes, and were analyzed and reported anonymously. No extramural funding was used to support this study.

One hundred and twenty-six out of 468 subjects had performed more than one PR program during the time period of the study. For the purposes of the study, we considered each subject/program as an individual case. ${ }^{15}$

\section{Pulmonary Function Tests and Arterial Blood Gas Analysis}

Vital capacity, $\mathrm{FEV}_{1}$, total lung capacity, and residual volume were measured by means of a flow-sensing spirometer and a body plethysmograph connected to a computer for data analysis (Masterlab, Erich Jaeger, Friedburg, Germany). Transfer factor of the lung for $\mathrm{CO}$ was measured by the single-breath method, using a mixture of carbon monoxide and methane (Sensor Medics, Yorba Linda, California). Vital capacity, $\mathrm{FEV}_{1}$, total lung capacity, residual volume, and transfer factor of the lung for $\mathrm{CO}$ were expressed as a percentage of the predicted values, which were obtained from regression equations by Quanjer et al ${ }^{16}$ and Cotes et al. ${ }^{17} \mathrm{FEV}_{1} /$ vital capacity and residual volume/total lung capacity ratios were taken as indices of airway obstruction and lung hyperinflation, respectively.

$\mathrm{P}_{\mathrm{aO}_{2}}$ and $\mathrm{P}_{\mathrm{aCO}}$ were measured immediately after sampling from a puncture of the radial artery at rest (ABL 330 gas analyzer, Radiometer, Copenhagen, Denmark).

\section{Dyspnea and Health Status by HRQOL}

Dyspnea was assessed by the baseline/transitional dyspnea index (BDI/TDI). ${ }^{18}$ BDI and TDI are intervieweradministered questionnaires composed of 3 categories (functional impairment, magnitude of task, and magnitude of effort). The BDI allows quantification of the limitation due to dyspnea at baseline, whereas TDI is useful to determine change from baseline level. In the BDI, each cat- egory has 5 levels of symptom severity, from 0 to 4 , where grade 0 corresponds to the most severe level. The category ratings are then summed to give a score, ranging from 0 to 12 , with a lower score indicating worse clinical condition. TDI evaluates change over time in each of the 3 categories. Change from baseline status is rated according to 7 grades, ranging from -3 (major deterioration) to +3 (major improvement), with 0 representing no change. The scores of the 3 categories are then summed to obtain the total score of the TDI, which ranges between -9 (a larger deterioration in dyspnea) and +9 . In our study, BDI/TDI were performed by a physiotherapist. HRQOL of subjects was evaluated by the VAS component of EQ-5D, reflecting their perceived health state, where 0 meant the worst imaginable health state and 100 meant the best imaginable health state. ${ }^{2}$

\section{Walking Capacity}

Walking capacity was evaluated by means of the distance covered during a 6MWT according to the ATS statement. ${ }^{19}$ The 6MWT was performed by all subjects in a 30-m indoor, level, hospital corridor, under the supervision of a physiotherapist, according to the ATS guidelines. All subjects received the same instructions before the walk, and were encouraged by the physiotherapist, who repeated set phrases every minute during the walk. A practice 6MWT was not performed. The 6-min walk distance (6MWD) covered during the test was recorded in meters. Subjects were allowed to stop and rest during the test but were instructed to resume walking as soon as they felt able to do so. In all subjects, the change in distance covered during 6MWT ( $\Delta 6 \mathrm{MWD})$ after PR was recorded. Before and immediately after the 6MWT, subjects rated the magnitude of their perceived breathlessness and of their leg fatigue using a $0-10$-point Borg scale.

\section{Pulmonary Rehabilitation Program}

Subjects underwent a comprehensive PR program consisting of: (1) exercise training, (2) unstructured programs for promoting self-management in COPD, (3) structured educational support, and (4) nutritional and psychological counseling, if needed. According to the guideline recommendations, the PR program was completely tailored to suit the needs of the individual. ${ }^{1}$ The program consisted of 15 sessions over a 3-wk period. ${ }^{20-23}$ To be included in the study, subjects had to have a frequency of at least 4 visits/wk. Lower limb endurance training was the main component of our PR program. All subjects performed 12 supervised sessions of $30-40 \mathrm{~min}$, using treadmill or cycle-ergometer, depending on the clinically based choice of the physiotherapist and on the subject preference. Exercise intensity was based on the initial $6 \mathrm{MWT},{ }^{24}$ and subjects 
started their training at $60-70 \%$ of the maximum heart rate achieved on the 6MWT. Exercises were then adjusted based on subject tolerance (at least weekly), with the aim of achieving a Borg dyspnea score of 3-5 (moderate to severe). To optimize training load, supplemental oxygen for subjects with chronic respiratory failure and interval training for very compromised subjects were adopted. Subjects who reported high impairment of their capacity in undertaking activities of daily living (climbing stairs and standing up from a low chair) also performed lower limb strength training on alternate days by sit-to-stand exercises. Each session also included supervised upper limb training; subjects used an arm ergometer or performed calisthenic exercises holding a light weight. In relation to the needs of subjects, the PR program could also include other components, such as pursed-lips breathing and exhalation on effort, as well as forward leaning position to improve diaphragm activity and optimize recruitment of accessory muscles of respiration, and inspiratory muscle training using threshold loading devices. Finally, each subject participated in educational activities, individually (at least 3 times) and in groups (at least 2 times), regarding selfmanagement, adherence to therapy, smoking cessation, and nutritional support. The total daily duration of activities was $2-3 \mathrm{~h}$, and the entire program was conducted in the hospital.

\section{Statistical Analysis}

This is a retrospective pilot study. Due to the explorative nature of the study, no formal sample size calculation was performed. Data are reported as mean $\pm \mathrm{SD}$, unless otherwise specified. The distribution of variables was assessed by means of Kolmogorov-Smirnov goodness-of-fit test. Relationships between variables were assessed by Pearson's correlation coefficient (r) and linear regression analysis. Comparisons between variables were determined by unpaired $t$ test and chi-square test, when appropriate. A statistical analysis by one-way analysis of variance was also performed to detect significant variations between groups, followed, where significant, by Student $t$ tests with Bonferroni adjustment.

To evaluate the MCID, the change in EQ-VAS score with PR was anchored against change in another outcome measure of PR efficacy. ${ }^{25}$ The receiver operating characteristic (ROC) curve method ${ }^{26}$ was therefore used to plot the true positive rate (sensitivity) in function of the false positive rate (1-specificity) for different cut-off points of EQ-VAS with respect to the MCID for TDI $(\geq 1), 27,28$ as a threshold value. The EQ-VAS cut-off point, which maximized sensitivity and specificity, was chosen as MCID.

The MCID for the EQ-VAS was then used to classify subjects with COPD into improved and unchanged categories.
Table 1. Baseline Characteristics of Subjects With COPD

\begin{tabular}{|c|c|c|c|c|}
\hline Characteristics & $\begin{array}{c}\text { All Subjects* } \\
(N=439)\end{array}$ & $\begin{array}{l}\text { Improved } \\
(n=314)\end{array}$ & $\begin{array}{c}\text { Unchanged } \\
(n=125)\end{array}$ & $P \dagger$ \\
\hline ge (y) & 7 & $70.7 \pm 7.9$ & $72 \pm$ & \\
\hline ender $(\mathrm{F} / \mathrm{M})$ & $/ 364$ & $54 / 260$ & $21 / 104$ & .92 \\
\hline $\mathrm{FEV}_{1}(\%$ predicted $)$ & $55 \pm 20$ & $51 \pm 17$ & $64 \pm 21$ & $<.001$ \\
\hline $\mathrm{VC}(\%$ predicted $)$ & 8 & $82=$ & 18 & $<.001$ \\
\hline $\mathrm{FEV}_{1} / \mathrm{VC}(\%)$ & $48 \pm 12$ & $46 \pm 12$ & $53 \pm 12$ & $<.001$ \\
\hline $\mathrm{RV}$ (\% predicted) & $169+45$ & 172 & 162 & .032 \\
\hline TLC (\% predicted) & 116 & 115 & 116 & .89 \\
\hline RV/TLC (\%) & 5 & 5 & 56 & $<.001$ \\
\hline IC (L) & $2.2=$ & $2.1=$ & 2.4 & $<.001$ \\
\hline redicted) & $61 \pm 22$ & $59 \pm 23$ & $66 \pm 21$ & .00 \\
\hline BMI $\left(\mathrm{kg} / \mathrm{m}^{2}\right)$ & $28.5 \pm 5.3$ & $28.7 \pm 5.7$ & $27.9 \pm 4.3$ & .16 \\
\hline $\mathrm{P}_{\mathrm{aO}_{2}}(\mathrm{~mm} \mathrm{Hg})$ & $70.6 \pm 8$ & $69.7 \pm 7.9$ & $73 \pm 7.8$ & $=.00$ \\
\hline $\mathrm{P}_{\mathrm{aCO}_{2}}(\mathrm{~mm} \mathrm{Hg})$ & $39.4 \pm 5.6$ & $40 \pm 5.7$ & $37.9 \pm 5$ & $<.001$ \\
\hline Charlson index & $1.5 \pm 1.3$ & $1.3 \pm 1.6$ & $1.5 \pm 1.3$ & .65 \\
\hline Exacerbations $(n)$ & $0.95 \pm 0.96$ & $1.03 \pm 0.97$ & $0.74 \pm 0.91$ & \\
\hline
\end{tabular}

Subjects with COPD were categorized as improved or unchanged, whether or not they

achieved the MCID in EQ-VAS ( $\geq 8$ ). Comparisons between variables were determined by unpaired $t$ test and chi-square test. Values are expressed as mean $\pm \mathrm{SD}$.

* All subjects who completed pre-pulmonary rehabiliation and post-pulmonary rehabiliation

evaluations and who were able to understand and produce visual analog scale scores.

$\dagger$ Improved vs unchanged.

$\mathrm{F}=$ female

$\mathrm{M}=$ male

$\mathrm{VC}=$ vital capacity

$\mathrm{RV}=$ residual volume

TLC $=$ total lung capacity

IC $=$ inspiratory capacity

$\mathrm{T}_{\mathrm{LCO}}=$ transfer factor of the lung for $\mathrm{CO}$

$\mathrm{BMI}=$ body mass index

A $P$ value $<0.05$ was considered as significant.

Data analyses and graphical presentations were performed using GraphPad Prism 5 (GraphPad, San Diego, California) or SPSS 20 (SPSS, Chicago, Illinois).

\section{Results}

According to GOLD criteria, 103 (22\%), 164 (35\%), 155 (33\%), and $46(10 \%)$ out of 468 subjects had, respectively, mild, moderate, severe, and very severe air flow obstruction. The mean number of visits during the 3-wk period of PR was $13 \pm 1$, and, in all subjects, the adherence rate to the PR program was $85 \%$ (range $80-100 \%$ ). There were 439 (94\%) subjects who were able to understand and produce EQ-VAS score. Characteristics of these subjects are reported in Table 1. At baseline, there was a ceiling effect for EQ-VAS in 4 of the subjects $(0.9 \%)$. After PR, these numbers increased to $9(2.1 \%)$. When subjects who underwent a single PR program were compared with those with more than 1 PR program, no difference was found in $\triangle \mathrm{EQ}-\mathrm{VAS}(15 \pm 12$ vs $14 \pm 11$, $P=.51)$.

All subjects showed a significant improvement in EQVAS, 6MWT, and TDI after PR. EQ-VAS improved by 


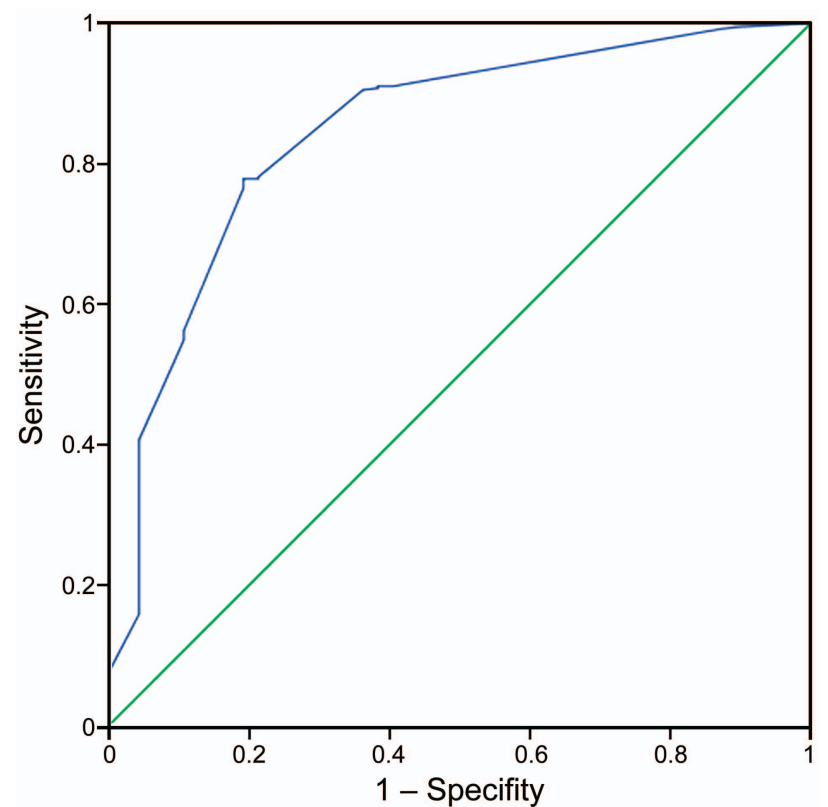

Fig. 1. Receiver operating characteristic curve analysis for different cutoff points of EQ-5D visual analog scale with respect to a minimal clinically important difference for transitional dyspnea index $(\geq 1)$. The area under curve value was $0.845(P<.001)$, and the cutoff point that maximized sensitivity $(0.78)$ and specificity (0.81) was a score of 8.

$14 \pm 12$ (from $58 \pm 17$ to $72 \pm 15, P<.001$ ). The $6 \mathrm{MWT}$ improved by $40 \pm 46 \mathrm{~m}$ (from $420 \pm 113$ to $460 \pm 103$, $P<.001)$. Dyspnea showed a clinically important reduction (from BDI $6.5 \pm 2.5$ to TDI $3.6 \pm 2.4$ ), corresponding to a change of $\geq 1$ unit in $89 \%$ of the subjects.

The ROC curve to identify the best change in EQ-VAS to discriminate between those achieving the MCID for TDI is shown in Figure 1. According to the ROC curve method, the plot of the true positive rate in function of the false positive rate for different cut-off points of EQ-VAS with respect to TDI $\geq 1$, as a threshold value, showed 0.845 area under curve value. The EQ-VAS cut-off point, which maximized sensitivity and specificity, was $\geq 8(0.78$ sensitivity and 0.81 specificity).

According to the proposed MCID for EQ-VAS, 314 out of 439 subjects $(72 \%)$ improved after PR, showing a EQVAS value $\geq 8$.

As compared with unchanged subjects in EQ-VAS score, the improved ones showed significantly worse respiratory function (air flow obstruction, hyperinflation, and diffuse lung capacity) and blood gas analysis (Table 1) and a higher percentage of frequent exacerbators ( $29 \%$ vs $16 \%$, $P=.004)$. Moreover, improved subjects obtained a higher change in 6MWD, TDI, dyspnea, and leg fatigue during 6MWT after PR than unchanged subjects (Table 2).
Table 2. Outcome Measures of PR in Subjects With COPD

\begin{tabular}{lcccc}
\hline \hline & $\begin{array}{c}\text { All } \\
\text { Subjects } \\
(n=439)\end{array}$ & $\begin{array}{c}\text { Improved } \\
(n=314)\end{array}$ & $\begin{array}{c}\text { Unchanged } \\
(n=125)\end{array}$ & $P^{*}$ \\
\hline EQ-VAS pre & $58 \pm 17$ & $55 \pm 15$ & $65 \pm 18$ & $<.001$ \\
EQ-VAS post & $72 \pm 15$ & $75 \pm 13$ & $66 \pm 18$ & $<.001$ \\
$\Delta$ EQ-VAS & $14 \pm 12$ & $19 \pm 9$ & $2 \pm 4$ & $<.001$ \\
6MWD pre & $420 \pm 113$ & $410 \pm 115$ & $445 \pm 103$ & .003 \\
6MWD post & $460 \pm 103$ & $461 \pm 102$ & $458 \pm 105$ & .81 \\
$\Delta 6$ MWD & $40 \pm 46$ & $51 \pm 47$ & $13 \pm 32$ & $<.001$ \\
BDI & $6.5 \pm 2.5$ & $6.1 \pm 2.4$ & $7.3 \pm 2.7$ & $<.001$ \\
TDI & $3.6 \pm 2.4$ & $4.4 \pm 2.2$ & $1.7 \pm 1.8$ & $<.001$ \\
Borg dyspnea pre & $3.8 \pm 1.9$ & $3.9 \pm 1.9$ & $3.5 \pm 1.8$ & .05 \\
Borg dyspnea post & $3.2 \pm 1.9$ & $3.1 \pm 1.8$ & $3.4 \pm 2.1$ & .23 \\
$\Delta$ Borg dyspnea & $0.6 \pm 1.7$ & $0.8 \pm 1.7$ & $0.2 \pm 1.8$ & $<.001$ \\
Borg fatigue pre & $3.4 \pm 1.9$ & $3.4 \pm 1.9$ & $3.5 \pm 2$ & .46 \\
Borg fatigue post & $2.9 \pm 2$ & $2.8 \pm 1.9$ & $3.4 \pm 2.1$ & .007 \\
$\Delta$ Borg fatigue & $0.5 \pm 2$ & $0.6 \pm 2$ & $0.1 \pm 2.1$ & .04
\end{tabular}

Subjects with COPD were categorized as improved or unchanged, whether or not they achieved the MCID in EQ-VAS $(\geq 8)$. Values are expressed as mean \pm SD. Comparisons between variables were determined by unpaired $t$ test and chi-square test. * Improved vs unchanged.

$\mathrm{PR}=$ pulmonary rehabilitation

EQ-VAS = EQ-5D visual analogue scale

pre $=$ before PR

post $=$ after PR

$\Delta=$ change after PR in outcome measures

$6 \mathrm{MWD}=6$-min walk distance

$\mathrm{BDI}=$ baseline dyspnea index

$\mathrm{TDI}=$ transitional dyspnea index

By analysis of variance, there were statistically significant differences in $\triangle E Q-V A S$ among subjects, categorized by the air flow obstruction degree $(8.2 \pm 10.3$, $14.7 \pm 10.6,16.1 \pm 11.6$, and $20.9 \pm 11.7$, respectively, $P<.001)$. Post hoc analyses showed statistically significant differences between subjects with mild obstruction and those of the other 3 stages, and between subjects with moderate obstruction and those with very severe obstruction $(P<.05$ for all comparisons).

Finally, in all subjects, $\triangle \mathrm{EQ}-\mathrm{VAS}$ was positively related to TDI $(\mathrm{r}=0.50, P<.001)$ and to change in $6 \mathrm{MWD}$ ( $\mathrm{r}=0.46, P<.001)$, whereas it was negatively related to baseline $\mathrm{FEV}_{1}(\mathrm{r}=-0.32, P<.001)$ (Table 3).

\section{Discussion}

In this retrospective study, we have investigated the role of a 3-wk PR program in determining benefits of EQ-VAS in 468 stable subjects with COPD. As expected, a significant improvement in EQ-VAS in all subjects was found. In addition, we have provided an MCID for EQ-VAS, able to discriminate improved from unchanged subjects with COPD, suggesting potential utility of this parameter in clinical settings. Furthermore, we found that improved subjects, as assessed by EQ-VAS, were characterized by worse 
Table 3. Correlations Observed With $\triangle$ EQ-VAS in All Subjects

\begin{tabular}{lc}
\hline \hline & $\Delta$ EQ-VAS \\
\hline TDI & $\mathrm{r}=0.50$ \\
& $P<.001$ \\
S6MWD & $\mathrm{r}=0.46$ \\
& $P<.001$ \\
FEV $_{1}(\%$ predicted $)$ & $\mathrm{r}=-0.32$ \\
& $P<.001$
\end{tabular}

Shown are the results of Pearson's correlation coefficient (r) when all subjects are considered. $\triangle \mathrm{EQ}-\mathrm{VAS}=$ change after pulmonary rehabilitation in EQ-5D visual analogue scale TDI $=$ transitional dyspnea index

$\triangle 6 \mathrm{MWD}=$ change after pulmonary rehabilitation in 6-min walk distance

respiratory function and higher changes in 6MWD and TDI after PR.

The effect of PR on EQ-5D in patients with COPD has not been adequately investigated. Up to now, only one study ${ }^{10}$ has examined this topic. In this study, Ringbaek et al ${ }^{10}$ analyzed 229 stable subjects with COPD before and after a 7-wk supervised PR program, by showing a significant improvement of EQ-5D. Notably, they showed the positive effect of PR only for EQ-5D utility score, whereas they observed a trend toward a significant increase in EQVAS score after PR $(P=.056) .{ }^{10}$ In the present study, we adopted an analogous number of PR program sessions in subjects with very similar EQ-VAS score at baseline to those of the Ringbaek et al study. ${ }^{10}$ Unlike the results in that study, our subjects experienced a significant improvement in EQ-VAS after PR. This discrepancy could be due to a different gender distribution. In the study by Ringbaek et al, ${ }^{10}$ there was a female predominance $(68 \%)$, whereas our study population was composed primarily of male subjects $(83 \%)$. It is known that there are gender-associated differences in HRQOL in subjects with COPD, showing poorer quality of life in women. ${ }^{29,30}$ Moreover, there is some evidence of lower improvements in HRQOL after PR in women with COPD, ${ }^{31}$ although the impact of gender-related differences in PR outcomes remains to be defined. ${ }^{32}$

As in the study by Ringbaek et al. ${ }^{10}$, we found a very small ceiling effect for EQ-VAS at baseline, with a slight increase after PR (from $0.9 \%$ to $2.1 \%$ ). Considering the simplicity and the quickness of EQ-VAS measure, we believe that the above values may be acceptable. In addition, floor and ceiling effects lower than 3\% were considered adequate for the St George Respiratory Questionnaire. ${ }^{33}$

Although EQ-5D is responsive to intervention and change (lung transplantation, self-management, tiotropium), ${ }^{34-36}$ there is a paucity of data regarding the minimal important difference. ${ }^{37,38}$ Walters et al $^{37}$ determined the minimal important difference for the EQ-5D from various datasets. Of the 11 studies that were reviewed, 1 involved subjects with COPD, ${ }^{11}$ considering only EQ-5D utility score but not EQ-VAS score. ${ }^{37}$ Notably, Pickard et al ${ }^{38}$ estimated the minimal important differences in EQ-5D utility and VAS scores in cancer. They conducted a retrospective analysis on data collected from 534 subjects with cancer, 50 of whom had lung cancer. Using both anchor-based and distribution-based approaches, Pickard et al ${ }^{38}$ observed that minimal important differences for VAS scores were similar for lung and all cancers, with a 7-point value.

In the present study, we provide the first indication of an MCID in EQ-VAS score after PR in subjects with COPD. Interestingly, compared with the results of Pickard et al ${ }^{38}$ in our study, we obtained a similar value of MCID. This fact supports the view that EQ-VAS could produce different scores for different medical conditions, but similar changes following interventions.

Furthermore, to evaluate the value of MCID for the EQ-VAS to distinguish improved and unchanged subjects, we divided subjects with COPD into 2 groups according to the MCID value. Interestingly, as compared with unchanged subjects, the improved ones showed significantly worse respiratory function (air flow obstruction, hyperinflation, and diffuse lung capacity) and blood gas analysis, and a higher percentage of exacerbators. Moreover, the improved group obtained a greater change in 6MWD, TDI, dyspnea, and leg fatigue during 6MWT after PR, than did the unchanged group. It is conceivable that subjects with poor baseline lung function are at risk to enter a downward spiral of dyspnea, sedentariness, demotivation, and finally deconditioning, ${ }^{39}$ as well as reporting significantly worse HRQOL, expressed by EQ-5D utility and VAS scores. ${ }^{4}$ On the other hand, our results showed that these subjects may have a larger improvement after PR, as compared with subjects with more preserved lung function, HRQOL, and exercise capacity.

Finally, we acknowledge that our population was partially composed of subjects who have performed more than one PR program during the time period of the study. This fact could influence the magnitude of the effects of PR on outcome measures, limiting the results of the study, in particular the change in EQ-VAS score. However, the effects of a PR program tend to decline toward baseline after 12 months, ${ }^{1}$ and its repetition confers similar increases in functional exercise capacity without providing additional effects. ${ }^{15}$ Similarly, in a previous $2-y$ controlled study, Foglio et $\mathrm{al}^{40}$ showed that successive, annual interventions lead to similar short-term gains but do not result in additive long-term physiologic benefits, except for the further reduction in the number of exacerbations. On the other hand, some additional physiological and clinical benefits were observed by Romagnoli et $\mathrm{al}^{41}$ in a study in which subjects repeated PR programs more frequently, that is, every 6 months. However, we did not observe a significant difference in EQ-VAS change between subjects with COPD that had undergone a single PR program 
as compared with those with more than one. Moreover, we acknowledge that the ROC curve method may not be the best way to identify MCID. However, up to now, there has been no consensus on the optimal method to estimate the MCID. ${ }^{42,43}$ Furthermore, the ROC curve method, using external anchor measures, was successfully used to calculate the MCID of the Clinical COPD Questionnaire ${ }^{44}$ and of the 5-repetition sit-to-stand test ${ }^{45}$ in subjects with COPD.

We acknowledge that the lack of control group may be a limitation of our study. A prospective randomized control group would add further consistency to the EQ-VAS cut-off point, as a MCID. PR still needs more evidence for its effectiveness in the medical community as well as to the insurance providers.

\section{Conclusions}

Our study shows good responsiveness of EQ-VAS score after PR. Moreover, even if the method used to calculate the MCID may not be optimal, we provide an MCID value for change in EQ-VAS score, in stable subjects with COPD. Furthermore, our results show that subjects with COPD with worse clinical and functional data seem to obtain more benefit from PR, with particular reference to change in EQ-VAS score. Although this assessment may not be exhaustive, the measurement of the quality of life through the VAS score represents a quick and easy evaluation for patients with COPD in a clinical setting.

\section{REFERENCES}

1. Spruit MA, Singh SJ, Garvey C, ZuWallack R, Nici L, Rochester C, et al. An official American Thoracic Society/European Respiratory Society statement: key concepts and advances in pulmonary rehabilitation. Am J Respir Crit Care Med 2013;188(8):e13-e64.

2. Brooks R, Rabin R, De Charro F, eds. The measurement and validation of health status using EQ-5D: a European perspective; evidence from the EuroQol BIOMED Research Programme, 1st edition. Dordrecht, The Netherlands: Kluwer Academic Publishers, 2003.

3. Ståhl E, Lindberg A, Jansson SA, Rönmark E, Svensson K, Andersson F, et al. Health-related quality of life is related to COPD disease severity. Health Qual Life Outcomes 2005;3:56.

4. Rutten-van Mölken MP, Oostenbrink JB, Tashkin DP, Burkhart D, Monz BU. Does quality of life of COPD patients as measured by the generic EuroQol five-dimension questionnaire differentiate between COPD severity stages? Chest 2006;130(4):1117-1128.

5. Pickard AS, Yang Y, Lee TA. Comparison of health-related quality of life measures in chronic obstructive pulmonary disease. Health Qual Life Outcomes 2011;9:26.

6. Wilke S, Janssen DJA, Wouters EFM, Schols JMGA, Franssen FME, Spruit MA. Correlations between disease-specific and generic health status questionnaires in patients with advanced COPD: a one-year observational study. Health Qual Life Outcomes 2012;10:98.

7. Menn P, Werber N, Holle R. Health-related quality of life in patients with severe COPD hospitalized for exacerbations: comparing EQ5D, SF-12 and SGRQ. Health Qual Life Outcomes 2010;8:39.

8. Goossens LM, Nivens MC, Sachs P, Monz BU, Rutten-van Mölken MP. Is the EQ-5D responsive to recovery from a moderate COPD exacerbation? Respir Med 2011;105(8):1195-1202.
9. Solem CT, Sun SX, Sudharshan L, Macahilig C, Katyal M, Gao X. Exacerbation-related impairment of quality of life and work productivity in severe and very severe chronic obstructive pulmonary disease. Int J Chron Obstruct Pulmon Dis 2013;8:641-652.

10. Ringbaek T, Brøndum E, Martinez G, Lange P. EuroQol in assessment of the effect of pulmonary rehabilitation COPD patients. Respir Med 2008;102(11):1563-1567.

11. Harper R, Brazier JE, Waterhouse JC, Walters SJ, Jones NM, Howard P. Comparison of outcome measures for patients with chronic obstructive pulmonary disease (COPD) in an outpatient setting. Tho$\operatorname{rax}$ 1997;52(10):879-887.

12. Zanini A, Chetta A, Gumiero F, Della Patrona S, Casale S, Zampogna E, et al. Six-minute walking distance improvement after pulmonary rehabilitation is associated with baseline lung function in clinically complex COPD patients: a retrospective study. Biomed Res Int 2013;2013:483162.

13. Global Initiative for Chronic Obstructive Lung Disease (GOLD). Global strategies for the diagnosis, management and prevention of chronic pulmonary disease. Updated 2014. http://www.goldcopd.org/ guidelines-global-strategy-for-diagnosis-management.html. Accessed September 18, 2014.

14. Charlson ME, Pompei P, Ales KL, MacKenzie CR. A new method of classifying prognostic comorbidity in longitudinal studies: development and validation. J Chronic Dis 1987;40(5):373-383.

15. Hill K, Bansal V, Brooks D, Goldstein RS. Repeat pulmonary rehabilitation programs confer similar increases in functional exercise capacity to initial programs. J Cardiopulm Rehabil Prev 2008;28(6): 410-414.

16. Quanjer PH, Tammeling GJ, Cotes JE, Pedersen OF, Peslin R, Yernault JC. Lung volumes and forced ventilatory flows. Report Working Party Standardization of Lung Function Tests, European Community for Steel and Coal. Official Statement of the European Respiratory Society. Eur Respir J Suppl 1993;16:5-40.

17. Cotes JE, Chinn DJ, Quanjer PH, Roca J, Yernault JC. Standardization of the measurement of transfer factor (diffusing capacity). Report Working Party Standardization of Lung Function Tests, European Community for Steel and Coal Official Statement of the European Respiratory Society. Eur Respir J Suppl 1993;16:41-52.

18. Mahler DA, Guyatt GH, Jones PW. Clinical measurements of dyspnoea. In: Mahler, DA. Dyspnoea. New York: Marcel Dekker; 1998.

19. ATS Committee on Proficiency Standards for Clinical Pulmonary Function Laboratories. ATS statement: guidelines for the six-minute walk test. Am J Respir Crit Care Med 2002;166(1):111-117.

20. Fuchs-Climent D, Le Gallais D, Varray A, Desplan J, Cadopi M, Préfaut C. Quality of life and exercise tolerance in chronic obstructive pulmonary disease: effects of a short and intensive inpatient rehabilitation program. Am J Phys Med Rehabil 1999;78(4):330335.

21. Clini E, Foglio K, Bianchi L, Porta R, Vitacca M, Ambrosino N. In-hospital short-term training program for patients with chronic airway obstruction. Chest 2001;120(5):1500-1505.

22. Cilione C, Lorenzi C, Dell Orso D, Garuti G, Rossi G, Totaro L, Clini E. Predictors of change in exercise capacity after comprehensive COPD inpatient rehabilitation. Med Sci Monit 2002;8(11): CR740-745.

23. Carone M, Patessio A, Ambrosino N, Baiardi P, Balbi B, Balzano G, et al. Efficacy of pulmonary rehabilitation in chronic respiratory failure (CRF) due to chronic obstructive pulmonary disease (COPD): the Maugeri study. Respir Med 2007;101(12):2447-2453.

24. Zainuldin MR, Knoke D, Mackey MG, Luxton N, Alison JA. Prescribing cycle training intensity from the six-minute walk test for patients with COPD. BMC Pulm Med 2007;7:9.

25. Make B. How can we assess outcomes of clinical trials: the MCID approach. COPD 2007;4(3):191-194. 


\section{EQ-5D Visual Analog Scale Score After Pulmonary Rehab}

26. Zweig MH, Campbell G. Receiver-operating characteristic (ROC) plots: a fundamental evaluation tool in clinical medicine. Clin Chem 1993;39(4):561-577.

27. Witek TJ Jr, Mahler DA. Minimal important difference of the transition dyspnoea index in a multinational clinical trial. Eur Respir $\mathrm{J}$ 2003;21(2):267-272.

28. Witek TJ Jr., Mahler DA. Meaningful effect size and patterns of response of the transition dyspnea index. J Clin Epidemiol 2003; 56(3):248-255.

29. de Torres JP, Casanova C, Hernández C, Abreu J, Aguirre-Jaime A, Celli BR. Gender and COPD in patients attending a pulmonary clinic. Chest 2005;128(4):2012-2016.

30. Naberan K, Azpeitia A, Cantoni J, Miravitlles M. Impairment of quality of life in women with chronic obstructive pulmonary disease. Respir Med 2012;106(3):367-373.

31. Foy CG, Rejeski WJ, Berry MJ, Zaccaro D, Woodard CM. Gender moderates the effects of exercise therapy on health-related quality of life among COPD patients. Chest 2001;119(1):70-76.

32. Robles PG, Brooks D, Goldstein R, Salbach N, Mathur S. Genderassociated differences in pulmonary rehabilitation outcomes in people with chronic obstructive pulmonary disease. J Cardiopulm Rehabil Prev 2014;34(2):87-97.

33. Barr JT, Schumacher GE, Freeman S, LeMoine M, Bakst AW, Jones $\mathrm{PW}$. American translation, modification, and validation of the St. George's Respiratory Questionnaire. Clin Ther 2000;22(9):1121-1145.

34. Al MJ, Koopmanschap MA, van Enckevort PJ, Geertsma A, van der Bij W, de Boer WJ, TenVergert EM. Cost-effectiveness of lung transplantation in the Netherlands. Chest 1998;113(1):124-130.

35. Monninkhof E, van der Valk P, Schermer T, van der Palen J, van Herwaarden C, Zielhuis G. Economic evaluation of a comprehensive self-management programme in patients with moderate to severe chronic obstructive pulmonary disease. Chron Respir Dis 2004;1(1): 7-16.
36. Covelli H, Bhattacharya S, Cassino C, Conoscenti C, Kesten S. Absence of electrocardiographic findings and improved function with once-daily tiotropium in patients with chronic obstructive pulmonary disease. Pharmacotherapy 2005;25(12):1708-1718.

37. Walters SJ, Brazier JE. Comparison of the minimally important difference for two health state utility measures: EQ-5D and SF-6D. Qual Life Res 2005;14(6):1523-1532.

38. Pickard AS, Neary MP, Cella D. Estimation of minimally important difference in EQ-5D utility and VAS scores in cancer. Health Qual Life Outcomes 2007;5:70.

39. MacIntyre NR. Mechanisms of functional loss in patients with chronic lung disease. Respir Care 2008 Sep;53(9):1177-1184.

40. Foglio K, Bianchi L, Ambrosino N. Is it really useful to repeat outpatient pulmonary rehabilitation programs in patients with chronic airway obstruction? A 2-year controlled study. Chest 2001;119(6): 1696-1704.

41. Romagnoli M, Dell'Orso D, Lorenzi C, Crisafulli E, Costi S, Lugli D, Clini EM. Repeated pulmonary rehabilitation in severe and disabled COPD patients. Respiration 2006;73(6):769-776.

42. Revicki D, Hays RD, Cella D, Sloan J. Recommended methods for determining responsiveness and minimally important differences for patient-reported outcomes. J Clin Epidemiol 2008;61(2):102-109.

43. Jones PW, Beeh KM, Chapman KR, Decramer M, Mahler DA, Wedzicha JA. Minimal clinically important differences in pharmacological trials. Am J Respir Crit Care Med 2014;189(3):250-255.

44. Kon SS, Dilaver D, Mittal M, Nolan CM, Clark AL, Canavan JL, et al. The Clinical COPD Questionnaire: response to pulmonary rehabilitation and minimal clinically important difference. Thorax. 2014; 69(9):793-820

45. Jones SE, Kon SS, Canavan JL, Patel MS, Clark AL, Nolan CM, et al. The five-repetition sit-to-stand test as a functional outcome measure in COPD. Thorax 2013;68(11):1015-1020. 